\title{
The neural basis for understanding imitation-induced musical meaning: the role of the human mirror system
}

Article

Accepted Version

Creative Commons: Attribution-Noncommercial-No Derivative Works 4.0

Jiang, J., Liu, F., Zhou, L. and Jiang, C. (2019) The neural basis for understanding imitation-induced musical meaning: the role of the human mirror system. Behavioural Brain Research, 359. pp. 362-369. ISSN 0166-4328 doi: https://doi.org/10.1016/j.bbr.2018.11.020 Available at https://centaur.reading.ac.uk/80601/

It is advisable to refer to the publisher's version if you intend to cite from the work. See Guidance on citing.

To link to this article DOI: http://dx.doi.org/10.1016/j.bbr.2018.11.020

Publisher: Elsevier

All outputs in CentAUR are protected by Intellectual Property Rights law, including copyright law. Copyright and IPR is retained by the creators or other copyright holders. Terms and conditions for use of this material are defined in the End User Agreement.

www.reading.ac.uk/centaur 
Central Archive at the University of Reading

Reading's research outputs online 
2 The neural basis for understanding imitation-induced musical

4

12 Correspondence should be addressed to:

13 Dr. Cunmei Jiang

14 Music College

15 Shanghai Normal University

$16 \quad 100$ E. Guilin Road

17 Shanghai, 200234, China

18 Tel: 0086-21-64322990; Fax: 0086-21-64322935

19 Electronic mail: cunmeijiang@126.com

20

21 


\section{Abstract}

2 Music can convey meanings by imitating phenomena of the extramusical world, and

3 these imitation-induced musical meanings can be understood by listeners. Although the

4 human mirror system (HMS) is implicated in imitation, little is known about the HMS's

5 role in making sense of meaning that derives from musical imitation. To answer this

6 question, we used fMRI to examine listeners' brain activities during the processing of

7 imitation-induced musical meaning with a cross-modal semantic priming paradigm. Eleven

8 normal individuals and 11 individuals with congenital amusia, a neurodevelopmental

9 disorder of musical processing, participated in the experiment. Target pictures with either an

10 upward or downward movement were primed by semantically congruent or incongruent

11 melodic sequences characterized by the direction of pitch change (upward or downward).

12 When contrasting the incongruent with the congruent condition between the two groups, we

13 found greater activations in the left supramarginal gyrus/inferior parietal lobule and inferior

14 frontal gyrus in normals but not in amusics. The implications of these findings in terms of

15 the role of the HMS in understanding imitation-induced musical meaning are discussed.

16

17 Keywords: fMRI, musical meaning, inferior frontal gyrus, inferior parietal lobule, 18 congenital amusia 


\section{$1 \quad 1$ Introduction}

2 Like language, music is unique to humans. Before language emerged, ancient human

3 ancestors were assumed to communicate with each other using gestures, facial expressions,

4 and songlike vocalizations [1], which imitated animal movements, animal calls, and sounds

5 of the natural world [2]. Being imitative in nature, musical vocalizations are also thought to

6 precede spoken words in human evolution [2-5]. A similar case has also been observed in

7 infant development, during which prelinguistic infants are able to make music-like sounds

8 that are imitations of maternal utterances [6,7]. Thus, imitation has been playing a critical

9 role in human communication, evolution, and development.

10 Similarly, people nowadays also convey intentions and meanings through music [8].

11 Musical meaning can emerge from the imitation of phenomena in the extramusical world,

12 including the sounds of creatures (e.g., birds), the qualities of objects (e.g., trains), and even

13 the representations of abstract concepts (e.g., wideness) [9-11]. As proposed by the mimesis

14 theory [12], music is an imitation or expression of the phenomena in our environment, and

15 this imitation or expression can be readily captured by listeners [13, 14]. Although

16 understanding the intentions of a composer or performer is a critical component in musical

17 meaning processing [15], little is known about the neural correlates underlying the

18 understanding of musical meaning that derives from the imitation of phenomena.

19 The human mirror system (HMS) consists of a core parietofrontal network of regions

20 in the inferior parietal lobule (IPL) and inferior frontal gyrus (IFG)/premotor cortex (PMC),

21 and it has been suggested that mirror neurons are fired during imitation of actions [16-19].

22 Substantial evidence has revealed that the HMS is activated by both action observation and

23 execution [20-24] and motor imagery [22, 25-27]. Listening to human action sounds, 
1 including speech sounds [28-31] and action-related sounds such as tearing paper, screaming

2 and kicking a football [32-35], also causes increased activations in the HMS. More

3 importantly, the HMS is also engaged in processing semantic information of action-related

4 sentences [36]. Taken together, previous studies indicate that imitation leads to the

5 activation of the HMS, where mirror neurons automatically map observed or heard actions

6 onto a motor program [37].

7 Although the HMS has also been assumed to be associated with imitation in music [38],

8 very few experimental studies have investigated this relationship directly. Previous studies

9 have shown that the HMS can be recruited by musical activities such as watching piano

10 performances [39, 40], tapping to rhythms [41], and making emotional judgments of songs

11 [42]. Furthermore, prior studies have also shown the engagement of a network of activity

12 resembling the HMS during reproducing melodies [43] and rhythmic patterns [44, 45].

13 However, these musical activities are irrelevant to music (or musical structure) per se.

14 Music itself can convey meanings by imitating the phenomena in the extramusical world [9-

15 11]. However, none of the previous studies has investigated the HMS's role in making sense

16 of imitation-induced musical meaning.

17 To address this issue, we used event-related fMRI techniques to investigate whether

18 the HMS is implicated in processing musical meaning derived from imitation. Using a

19 cross-modal semantic priming paradigm, a widely used design for studies of meaning

20 processing in music [e.g., 46, 47], we presented participants with melodic sequences

21 (primes) that were followed by pictures (targets). The melodic sequences contained a series

22 of notes with either an upward or a downward direction, and the target pictures portrayed

23 either a simple upward or downward movement, with the effect of picture complexity on 
1 brain activity controlled for [48]. Thus, the target pictures were either semantically

2 congruent or incongruent to the musical primes. We hypothesized that, if the listeners were

3 able to process the semantic relationship between the primes and targets by showing a

4 priming effect, it would indicate that they had understood the link between the musical

5 imitation (through melodic sequences) and the natural phenomena (as depicted by the

6 pictures).

7 Apart from normal participants, we also tested individuals with congenital amusia

8 (hereafter amusia), a neurodevelopmental disorder of musical processing [49]. Individuals

9 with amusia not only have difficulty discriminating fine-grained pitch differences [50-53],

10 but also show deficits in perceiving pitch direction $[50,51,54,55]$ and melodic contour [ $[51$,

11 56], which has led to the "melodic contour deafness hypothesis" by Patel [57]. Given that

12 melodic contour is a building block for musical melodies, deficits in melodic contour

13 perception may affect the understanding of musical meaning in amusia. Indeed, our

14 previous study showed that amusics failed to elicit an N400 effect to musical meaning

15 represented by the direction of pitch change, even though the pitch distances between the

16 first and last notes of the melodic sequences exceeded their pitch direction discrimination

17 thresholds [47]. Thus, by including both normal and amusic individuals as participants, we

18 would be able to reveal the core brain regions that are necessarily required for effective

19 processing of musical meaning, as compared to dysfunctional processing in amusia. Given

20 the role of the HMS in imitation [e.g., 16, 18], we predicted that the parietofrontal regions

21 including the IPL and IFG/PMC would be activated during the understanding of musical

22 meaning in normal but not in amusic individuals. 


\section{Materials and methods}

\section{$2 \quad 2.1$ Participants}

3 Twelve amusic and 12 normal individuals took part in the experiment. The Montreal

4 Battery of Evaluation of Amusia (MBEA), consisting of six subtests - scale, contour,

5 interval, rhythm, meter, and memory, was used to assess musical abilities of these

6 participants $[58,59]$. Participants were identified as amusics if they scored 65 or below on

7 the melodic composite score (sum of the scores on the scale, contour, and interval tests)

8 [54], and below 78\% correct on the MBEA global score [59]. Furthermore, participants'

9 pitch change detection and pitch direction discrimination thresholds were measured using a

10 two-alternative forced choice AXB paradigm [50], in which a sequence of three stimuli (A,

$11 \mathrm{X}$, and $\mathrm{B}$ ) were presented in each trial and the second one (X) was either the same as the

12 first (A) or last (B). In particular, on each trial of the pitch change detection threshold task,

13 three pure tones (A, X, and B) were presented consecutively, among which tone $\mathrm{X}$ was

14 identical to either tone A or B. Participants were required to judge whether tone $\mathrm{X}$ had the

15 same pitch as tone A or B. On each trial of the pitch direction discrimination threshold task,

16 three 2-tone pairs (A, X, and B) were presented, where pair X was identical in pitch

17 direction to either pair A or B. Participants were required to indicate whether the pitch

18 direction of pair X was the same as that of pair A or B. Experimental trials were presented

19 with an adaptive staircase procedure, in which the initial pitch difference was four

20 semitones and was then varied with a "two-down, one-up" staircase procedure. The

21 procedure terminated after 16 reversals, and the threshold was computed based on the

22 average pitch difference of the last six reversals. For more details please refer to ref. [50].

23 After the fMRI experiment, one amusic participant and one matched control participant 
1 were excluded from further analysis based on a head motion criterion of $3 \mathrm{~mm}$ of

2 translational and $3^{\circ}$ of rotational movement across the session. As a result, the final sample

3 consisted of 11 participants in each group.

4 As shown in Table 1, the two groups were matched in age, sex, and education, but the

5 amusic group showed worse performance on the MBEA and higher pitch thresholds than

6 the control group. All participants were right-handed as assessed by the Edinburgh

7 Handedness Inventory [60]. All had normal hearing and reported to have normal or

8 corrected-to-normal vision. None had reported history of neurological or psychiatric

9 diseases. None of the controls received formal musical training or played any musical

10 instruments. One amusic participant had one year of singing lessons and another had one

11 year of piano lessons. Ethical approval was granted by Shanghai Normal University in

12 China, and all participants gave written informed consent and were paid for their

13 participation.

14

15 Table 1 Characteristics of participants

\begin{tabular}{lcccc}
\hline Variable & Amusics & Controls & Group-statistic & $d(95 \% \mathrm{CI})$ \\
\hline Demographic variable & & & & \\
Age in years & $22.91(2.55)$ & $23.09(0.94)$ & $t(12.69)=-0.22, p=.828$ & $-0.09(-0.93,0.74)$ \\
Sex (male: female) & $3: 8$ & $3: 8$ & - & - \\
Handedness & $11 \mathrm{R}$ & $11 \mathrm{R}$ & - & $-0.43(-1.27,0.43)$ \\
Education in years & $15.64(2.11)$ & $16.36(1.12)$ & $t(15.22)=-1.01, p=.329$ & \\
Mean scores of MBEA & & & & \\
Scale & $18.27(2.72)$ & $27.91(1.22)$ & $t(13.86)=-10.71, p<.001$ & $-4.57(-6.43,-2.67)$ \\
Contour & $19.00(3.29)$ & $28.27(0.90)$ & $t(11.51)=-9.02, p<.001$ & $-3.85(-5.59,-2.07)$
\end{tabular}




\begin{tabular}{|c|c|c|c|c|}
\hline Interval & $18.00(3.90)$ & $27.64(1.50)$ & $t(12.90)=-7.65, p<.001$ & $-3.26(-4.74,-1.74)$ \\
\hline Rhythm & $19.73(3.88)$ & $26.91(1.64)$ & $t(13.47)=-5.66, p<.001$ & $-2.41(-3.62,-1.16)$ \\
\hline Meter & $18.45(5.05)$ & $27.18(2.79)$ & $t(20)=-5.02, p<.001$ & $-2.14(-3.19,-1.06)$ \\
\hline Memory & $20.36(5.08)$ & $28.00(1.48)$ & $t(11.69)=-4.78, p<.001$ & $-2.04(-3.19,-0.85)$ \\
\hline Melodic score & $55.27(7.21)$ & $83.82(2.86)$ & $t(13.06)=-12.20, p<.001$ & $-5.20(-7.33,-3.05)$ \\
\hline Global score (\%) & $63.23(5.90)$ & $92.17(3.10)$ & $t(15.12)=-14.40, p<.001$ & $-6.14(-8.45,-3.81)$ \\
\hline
\end{tabular}

Pitch perception threshold

Pitch change detection

$1.40(1.04) \quad 0.27(0.12) \quad t(10.27)=3.57, p=.005$

$1.52(0.44,2.56)$

Pitch direction discrimination

$4.02(1.32)$

$0.48(0.22)$

$t(10.57)=8.76, p<.001$

$3.74(1.94,5.49)$

Note. $\mathrm{R}=$ right-handed; $\mathrm{MBEA}=$ Montreal Battery of Evaluation of Amusia. MBEA scores are expressed as the number of correct responses out of 30. Pitch perception thresholds are

3 in semitones. Standard deviation values are shown in parentheses.

\section{$5 \quad 2.2$ Stimuli}

A total of 84 short melodic sequences were created as potential prime stimuli, which

7 consisted of 15 quarter notes with either an upward or a downward pitch direction. Half of

8 the sequences were constructed with a small pitch distance no more than two semitones

9 between two consecutive tones, whereas the other half were constructed with a large pitch

10 distance of more than four semitones. Because all sequences had an ascending or a

11 descending direction, the pitch distances between the first and last tones ranged from 12 to

1228 semitones. Each sequence lasted four seconds. All notes were generated by Overture 4.1

13 (GenieSoft Inc.) and played with a grand piano sound using Pianissimo 1.0 (Acoustica Inc.).

14 Using Adobe Audition CS6 (Adobe Systems Inc.), these sequences were normalized to have

15 the same sound intensity level $(-3 \mathrm{~dB})$ and $400 \mathrm{~ms}$ fade-out ramps. 
Target pictures were two schematic representations, one with an upward movement

2 and the other with a downward movement. Each prime was paired with a target picture to

3 form semantically congruent or incongruent conditions (see Fig. 1), thus resulting in 168

4 prime-target pairs.

5

6

7

8

9 ratings on 48 incongruent pairs: $M=2.84, S D=0.60 ; t(47)=31.58, p<.001$ ) were selected

17 for the fMRI experiment. Among these pairs, primes comprised of 24 upward and 24 downward melodic sequences with 12 small and 12 large pitch distances, respectively. As

19 in the pretest, each prime was used twice: in one trial, the prime was semantically congruent

20 to the target picture; and in the other trial, the prime and target were incongruent.

\section{$21 \quad 2.3$ Procedure}

Before performing the fMRI experiment, participants were first trained on the task

23 using a personal computer. They repeatedly practiced six trials outside the scanner until 
1 they became fully familiarized with the task. Inside the scanner, foam pads were used, and

2 explicit verbal instructions were given to the participants in order to limit their head motion.

3 Using the E-prime 2.0 software (Psychology Software Tools Inc.), visual stimuli were

4 presented and projected onto a screen at the front of the scanner. Participants viewed these

5 stimuli through a mirror attached to the head coil. Auditory stimuli were presented via MR-

6 compatible headphones. Participants wore foam earplugs to reduce the effect of ambient

7 scanner noise. Before scanning, the sound volume was adjusted to a comfortable listening

8 level for each participant.

9 During the scanning session, participants were asked to keep their body still and their

10 eyes open, directing toward the middle of the screen. They were first given the same six

11 trials for practice. Each trial started with a black fixation cross (with a random duration of 2

$12 \mathrm{~s}, 4 \mathrm{~s}$, or $6 \mathrm{~s})$ at the center of the white screen. When the fixation cross disappeared, the

13 prime was presented with a black screen background ( $4 \mathrm{~s})$, followed by the visual

14 presentation of a target picture $(2 \mathrm{~s})$. After the target picture had disappeared, a response

15 screen $(2 \mathrm{~s})$ appeared and participants were asked to indicate whether the meaning

16 expressed by the prime-target pair was congruent or incongruent by pressing one of the two

17 response buttons with their thumbs. Half of the participants responded to the congruent

18 trials with the right thumb and the incongruent trials with the left thumb, and the other half

19 did the opposite. The next trial began $2 \mathrm{~s}$ after the previous trial, no matter whether the

20 participants made their response within $2 \mathrm{~s}$ or not. The 96 trials were presented in pseudo-

21 randomized order with a maximum of three congruent or incongruent trials in a row. These

22 trials were divided into two sessions with a break in between. The order of presentation was

23 counterbalanced across participants. 


\section{$1 \quad 2.4$ Data acquisition and analysis}

2 Participants were scanned on a 3T Siemens Magnetom Trio Tim System (Siemens,

3 Erlangen, Germany) with a 12-channel head coil. Functional images were acquired using a

4 gradient echo-planar imaging sequence with the following parameters: repetition time $=2 \mathrm{~s}$,

5 echo time $=30 \mathrm{~ms}$, field of view $=192 \mathrm{~mm}$, flip angle $=90^{\circ}$, matrix size $=64 \times 64 \mathrm{~mm}$,

6 voxel size $=3 \times 3 \times 3 \mathrm{~mm}^{3}, 37$ axial slices, slice thickness $=3 \mathrm{~mm}$, and inter-slice gap $=0.6$

$7 \mathrm{~mm}$.

8 The analysis of the fMRI data was performed using the DPABI 3.0 [61] and SPM 12

9 toolboxes (Wellcome Trust Center for Neuroimaging, London, UK) run in Matlab R2016a

10 (MathWorks Inc, Natick, MA, USA). The two toolboxes offer complementary functions.

11 Compared to SPM, DPABI requires less manual operations, making preprocessing less

12 time-consuming with a reduced risk of inadvertent mistakes [61, 62]. However, SPM, but

13 not DPABI, provides further statistical analyses (e.g., GLM analysis) for task-based fMRI

14 data [61]. Since DPABI also calls SPM functions in preprocessing data [62], it is convenient

15 to perform the GLM analysis using SPM based on the preprocessed data by DPABI [61].

16 Therefore, the functional imaging data were first preprocessed in DPABI. For preprocessing,

17 blood oxygen level dependent (BOLD) images were realigned to the first functional image

18 in order to correct for head movement. The realigned volumes were normalized to the EPI

19 template in the Montreal Neurological Institute (MNI) space. Each normalized image was

20 then smoothed with a 6-mm full-width at half-maximum isotropic Gaussian kernel.

21 Statistical analyses based on the general linear model were then carried out in SPM. At the

22 first level, neural activity during the presentation of the target was modeled by a canonical

23 hemodynamic response function and its temporal derivative. For each participant, four 
1 conditions were modeled separately: congruent trials with correct responses, congruent

2 trials with incorrect and omitted responses, incongruent trials with correct responses,

3 incongruent trials with incorrect and omitted responses. Additionally, we included six

4 regressors modeling head movement parameters. To reduce low frequency drifts, a high-

5 pass filter was set at $128 \mathrm{~s}$. An autoregressive AR(1) model was applied to account for

6 temporal autocorrelation intrinsic to the fMRI time-series. Individual contrast images for the

7 conditions "incongruent against congruent trials with correct responses" and "congruent

8 against incongruent trials with correct responses" were generated. One-sample $t$-tests were

9 performed to evaluate whether the observed differences were significantly different from

10 zero within each group separately. Regions consisting of a cluster size of at least 10 voxels

11 that exceeded an uncorrected voxel threshold of $p<.001$ were considered reliable. At the

12 second level, two-sample independent $t$-tests were used to investigate the effect of group.

13 Considering the role of the HMS in imitation, we included the combined regions of interest

14 (ROIs; i.e., the bilateral IPL, supramarginal gyrus, IFG, and precentral gyrus) for small

15 volume correction analysis. All anatomical ROIs were constructed using the Talairach

16 Daemon option of the WFU PickAtlas 3.0.5 toolbox [63]. Statistical significance was set at

17 voxel-level uncorrected $p<.001$ and cluster-level false discover rate (FDR) corrected $p$

$18<.05$ with $k>10$ voxels. Anatomical labels for activated regions were provided by the SPM

19 Anatomy toolbox version 2.2c $[64,65]$ and if unavailable, then provided by the xjView 9.6

20 toolbox (http://www.alivelearn.net/xjview/).

21 Bivariate correlations were computed between MBEA scores, behavioral data, and beta

22 values of the ROIs. For each ROI demonstrating a significant group effect, the MarsBaR

230.44 toolbox (http://marsbar.sourceforge.net/index.html) was used to extract each 
1 participant's mean BOLD parameter estimate value (beta value) from a $6 \mathrm{~mm}$ radius sphere

2 around peak activations.

\section{$3 \quad 3$ Results}

\section{$4 \quad 3.1$ Behavioral results}

5 To eliminate the contribution of response bias, we adopted the sensitivity measure

6 from the signal detection theory [66] as an accuracy index. A "yes" response to a congruent

7 trial was defined as a hit whereas a "yes" response to an incongruent trial was defined as a

8 false alarm. To avoid the biasing effect of extreme hit or false-alarm proportions of zero or

9 one on $d^{\prime}$ values, we adopted the strategy of adding 0.5 to all data cells in the two-by-two

10 contingency table $[67,68]$.

11 The values of $d^{\prime}$ were submitted to a two-way repeated-measures ANOVA with group

12 (amusics, controls) as a between-subjects factor and pitch direction (upward, downward) as

13 a within-subjects factor. Neither the main effect of pitch direction, $F(1,20)=0.92, p=.349$,

$14 \eta_{\mathrm{p}}^{2}=.04$, nor the interaction between group and pitch direction, $F(1,20)=1.15, p=.296$,

$15 \eta_{\mathrm{p}}{ }^{2}=.05$, was significant. Therefore, the data were collapsed across pitch direction. The

16 two-sample $t$-test showed that controls $(M=2.84, S D=0.99)$ performed better than amusics

$17(M=0.85, S D=0.77), t(20)=-5.26, p<.001, d=-2.24,95 \%$ CI $[-3.31,-1.14]$, on judging

18 semantic congruency between the primes and targets.

\section{$19 \quad 3.2$ Neuroimaging results}

20 As can be seen in Table 2, when comparing incongruent with congruent trials, amusics

21 showed more activation in the left extra-nuclear and right sub-gyral, caudate nucleus, and

22 the brainstem. Controls, by contrast, only showed increased activation in the left

23 supplementary motor area (Boersma, \#3817). SMA is a relevant area with regard to 
1 sensorimotor interactions. A possible interpretation for this activation is that it may be a

2 stimulus driven motor representation rather than an intention driven representation.

4 Table 2 Differential brain activations from the within-group and between-group analyses

5 (incongruent $>$ congruent)

\begin{tabular}{|c|c|c|c|c|c|c|}
\hline \multirow[b]{2}{*}{ Region of activation } & \multirow[b]{2}{*}{$\mathrm{BA}$} & \multirow[b]{2}{*}{ No. of voxels } & \multicolumn{3}{|c|}{ MNI coordinates } & \multirow[b]{2}{*}{$z$ score } \\
\hline & & & $x$ & $y$ & $z$ & \\
\hline \multicolumn{7}{|l|}{ Amusics } \\
\hline R Sub-gyral & & 20 & 24 & -18 & 27 & 3.91 \\
\hline R Caudate nucleus & & & 18 & -18 & 21 & 3.63 \\
\hline L Extra-nuclear & & 10 & -6 & -36 & 3 & 3.69 \\
\hline R Brainstem & & & 3 & -33 & 0 & 3.49 \\
\hline \multicolumn{7}{|l|}{ Controls } \\
\hline L Supplementary motor area & 6 & 11 & -3 & 3 & 57 & 4.01 \\
\hline \multicolumn{7}{|l|}{ Controls $>$ Amusics } \\
\hline L Supramarginal gyrus* & 40 & 19 & -48 & -45 & 30 & 4.05 \\
\hline L Inferior frontal gyrus* & 45 & 21 & -48 & 27 & 15 & 3.70 \\
\hline L Inferior frontal gyrus* & & & -51 & 33 & 12 & 3.50 \\
\hline \multicolumn{7}{|l|}{ Amusics $>$ Controls } \\
\hline no significant clusters & & & & & & \\
\hline
\end{tabular}

$6 \quad$ Note. $\mathrm{L}=$ left; $\mathrm{R}=$ right. * Small volume-corrected $p \mathrm{~s}=.036$ (FDR corrected).

7

8 When comparing the incongruent $>$ congruent contrast between groups, we found that

9 controls yielded a greater activation in the left supramarginal gyrus/IPL and IFG than

10 amusics (see Fig. 2). No activated region was found in amusics relative to controls. 
Insert Figure 2, about here.

4 The results from the opposite comparison of congruent $>$ incongruent trials are

5 presented in Table 3. For the within-group analysis, neither amusics nor controls showed

6 more clusters for congruent than incongruent trials. These results may be explained by

7 fMRI adaptation, according to which unrelated stimuli elicit enhanced neural activation

8 compared to related stimuli while related stimuli reduce BOLD responses relative to

9 unrelated or novel stimuli $[69,70]$. For the between-group analysis, amusics showed more

10 clusters in the left IPL and IFG for congruent than incongruent trials compared to controls.

11 In other words, amusics showed reduced BOLD responses to incongruent trials in the left

12 IPL and IFG, while controls demonstrated increased responses to incongruent trials in these

13 regions. These results were consistent with those obtained by comparing incongruent with

14 congruent trials in Table 2.

15

16 Table 3 Differential brain activations from the within-group and between-group analyses

17 (congruent $>$ incongruent)

MNI coordinates

Region of activation $\quad$ BA $\quad$ No. of voxels $\begin{array}{lll}x & y & z\end{array}$ score

\section{Amusics}

no significant clusters

\section{Controls}

no significant clusters

\section{Controls $>$ Amusics}

no significant clusters 


\section{Amusics $>$ Controls}

$\begin{array}{lllllll}\text { L Supramarginal gyrus* } & 40 & 19 & -48 & -45 & 30 & 4.05 \\ \text { L Inferior frontal gyrus* } & 45 & 21 & -48 & 27 & 15 & 3.70 \\ \text { L Inferior frontal gyrus* } & & & -51 & 33 & 12 & 3.50\end{array}$

1 Note. $\mathrm{L}=$ left; $\mathrm{R}=$ right. $*$ Small volume-corrected $p \mathrm{~s}=.036$ (FDR corrected).

3 We also analyzed the correlations among MBEA scores, accuracy in congruency

4 judgment and the associated brain activations, but not the correlations between pitch

5 thresholds and these measures, because the pitch distances between the first and last notes

6 of the melodic sequences exceeded participants' thresholds for pitch perception. For

7 amusics, neither the MBEA scores nor the behavioral measures of performance were

8 significantly correlated with the beta values of the IPL $(p s>.555)$ or IFG $(p s>.504)$.

9 Similarly, none of the correlations was significant for controls (all $p \mathrm{~s}>.200$ ). The lack of

10 correlation between these measures might be because we had a limited sample of

11 participants with homogeneous characteristics, which might have underestimated the true

12 degree of relationship between these variables $[71,72]$. When the data from the two groups

13 were pooled, all correlations were significantly positive $(r(20)>.44, p \mathrm{~s}<.042)$, due to the

14 between-group differences.

\section{Discussion}

16 Using fMRI, we investigated the neural correlates underlying the understanding of

17 musical meaning that derives from the imitation of natural phenomena in our environment.

18 While amusic individuals showed a dysfunctional HMS in the understanding of musical

19 meaning, normal individuals showed significantly increased activations in the left IPL and 
1 the left IFG during comprehension of musical meaning. These findings reveal for the first

2 time the HMS as the prime agent for understanding musical meaning that derives from

3 imitation and extend its role to abstract representation of meaning in music.

4 The first main finding of our study is that the left HMS is activated in normal

5 individuals during the understanding of musical meaning that derives from the imitation of

6 phenomena. In the HMS, the IPL is mostly implicated in motoric descriptions of actions [19,

7 20, 73], while the IFG is more concerned with the goal or intention of actions [74-76]. In

8 our study, when listening to an upward or a downward melody, the listeners needed to map

9 the kinematic pattern of the heard pitch movement onto an internal motor representation of

10 the same movement. Consistent with the IPL's role in sensorimotor transformation [77-79],

11 we observed stronger BOLD responses to incongruent than congruent trials in the IPL. In

12 addition, the ascending and descending melodic sequences in the present study cued the

13 intentions of upward and downward movements (e.g., going upstairs or downstairs). To

14 judge the semantic congruency between the picture and the melody, participants had to infer

15 the intentions of the movements of the primes and targets. Therefore, the activation of the

16 IFG in normal individuals reflected the understanding of these intentions.

17 Although the PMC is often activated during imitation [23, 80-82], no significant

18 activation in the PMC was observed in our study. However, after using a more liberal

19 threshold of uncorrected $p<.001$ and cluster size of $k=0$ voxels, we observed activity

20 changes in the middle frontal gyrus $(x=-48, y=6, z=54, z$ score $=3.37, p<.001, k=1$,

21 BA 6$)$ and supplementary motor area $(x=-3, y=6, z=57, z$ score $=3.26, p=.001, k=2$,

22 BA 6). There are two possible interpretations for the less robust activation of the PMC in

23 the present study. One possibility may lie in the fact that our participants were not familiar 
1 with the experimental stimuli and task. Indeed, previous studies have reported that the PMC

2 and supplementary motor area are significantly more active for familiar than unfamiliar

3 stimuli, such as music [83-86] and visual pictures [87, 88]. In the present study, although

4 walking up or down the stairs as depicted in the target pictures could be considered as

5 familiar actions, the melodic sequences that were composed specifically for the current

6 experiment could be unfamiliar to our participants. This might have resulted in the

7 nonsignificant activation of the PMC in our study. Future studies are required to further

8 explore this possibility. Another possibility is related to the modality of stimulus

9 presentation. It has been suggested that the premotor activity is based on unimodal (visual,

10 auditory, or tactile) attention shifts [89-92], which are tightly coupled to the motor program

11 [93]. However, our study involved crossmodal attention shifts from auditory cues to visual

12 targets, which resulted in decreased activation of the PMC. Indeed, behavioral research has

13 confirmed that the processing of unimodal auditory cues and targets is coupled to the motor

14 program, which is not the case for crossmodal auditory cues and visual targets [94].

15 The activation of the HMS is confined to the left hemisphere in our study, which is

16 consistent with previous evidence that action sounds are associated with the activation of

17 the left HMS $[32,95,96]$. However, it is contradictory to the finding that bilateral HMS is

18 involved during action observation and imitation [21,97]. This discrepancy may be

19 attributed to the difference in stimuli across different studies. The stimuli used in the present

20 study were both auditory and visual stimuli (i.e., the musical primes and target pictures),

21 whereas the stimuli in those previous studies $[21,97]$ were visual stimuli only. It has been

22 shown that the left hemisphere of the human brain has a multimodal (visual, auditory) HMS

23 [19] and may code actions at a more abstract level or in an amodal manner [98], while the 
1 right hemisphere only has a visual HMS [19]. Thus, the activation of the left HMS in our

2 study may reflect the multimodal (visual, auditory) stimuli used.

3 The second main finding of our study is that the HMS is involved in the abstract

4 representation of musical meaning. Previous fMRI data have indicated that the HMS is

5 implicated in the processing of action-related information, such as executing actions [22],

6 perceiving actions [e.g., 23, 28, 35], and imagining actions [e.g., 22, 25]. Some evidence

7 also suggests that a mirror-like system is implicated in producing musical stimuli [43-45].

8 Our study extends these results by suggesting the role of the HMS in understanding

9 meaning in music. Particularly, in the present study, each melody with an upward or a

10 downward pitch direction was paired with a picture with an upward movement and another

11 with a downward movement. In order to judge whether the meaning of the target picture

12 was congruent or incongruent with the meaning of the prime, the participants had to extract

13 the meanings from both the prime and the target. Thus, the activation of the HMS reflected

14 the extraction of abstract meaning. On the other hand, what we manipulated was the

15 semantic relations between musical primes and target pictures. This design ruled out the

16 possibility that the HMS was activated by musical primes or target pictures alone. Indeed,

17 the IPL and IFG share cognitive functions, especially in semantic integration [99-101],

18 possibly due to the anatomical connections between the two [102, 103]. Consequently, the

19 activation of the HMS reflected semantic integration processes. In short, the HMS in the

20 present study is involved in the understanding of abstract meaning in music.

21 The third main finding of our study is that unlike normals, amusics demonstrated a

22 dysfunctional HMS in the understanding of musical meaning, which is in agreement with

23 our prior EEG study that reported the absence of an N400 effect in processing musical 
1 meaning in amusia [47]. The failure to derive musical meaning from pitch change direction

2 confirmed that amusia is indeed a pitch-related disorder, including pitch perception [e.g., 50,

3 51, 104], tonal functional cognition [105-107], and emotion perception [108-111]. Although

4 impaired pitch discrimination and memory in amusia is related to abnormal activity changes

5 in the right IFG as well as decreased functional connectivity between the right IFG and the

6 right auditory cortex [112-114], our study further indicates that impaired musical meaning

7 understanding in amusia is associated with aberrant brain activity in the left parietofrontal

8 cortices. These results imply that functional anomalies in the amusic brain may depend on

9 task demands. Moreover, given that the HMS is an innate endowment [e.g., 115, 116], our

10 findings confirm the assumption that the pitch-based deficit of amusia is likely to be present

11 at birth $[117,118]$. Indeed, it has been suggested that amusia has a genetic basis $[119,120]$.

12 From this perspective, one could speculate that the brain function for understanding musical

13 imitation may be hardwired in the brain of neurotypical individuals, but not in that of

14 amusic individuals.

15 In sum, the present data show that the HMS plays an important role in understanding

16 musical meaning that derives from the imitation of phenomena, providing new insight into

17 the neural representation of musical meaning in the HMS. It is worth noting that we used

18 the direction of pitch change to imitate an upward or a downward movement, which reflects

19 a creative intention. Interestingly, Steinbeis and Koelsch [15] manipulated experimental

20 instructions (that is, whether participants were informed that the piece of music was written

21 by a composer or generated by a computer), and found that understanding the composer's

22 intentions activated the anterior medial frontal cortex, superior temporal sulcus/middle

23 temporal gyrus/IPL, temporal pole, IFG, and middle occipital gyrus in listeners. Therefore, 
1 it is reasonable to assume that the HMS may be involved in the understanding of musical

2 meaning, regardless of whether they are derived from imitation or not. Taken together, our

3 findings provide functional imaging evidence in support of the mimesis theory that music is

4 an imitation of phenomena, and imitation-induced musical meaning can be readily

5 understood by normal listeners [12-14]. 


\section{Acknowledgements}

2 The authors thank Dr. Lisa Aziz-Zadeh, Xiaochen Tang, Dr. Haijiang Li, and Dr.

3 Shengchuang Feng for their valuable suggestions on an earlier version of this manuscript.

4 This work was supported by the National Natural Science Foundation of China (grant

5 number 31470972 to CJ and FL, and grant number 31500876 to LZ and JJ.), and the

6 European Research Council Starting Grant (CAASD, No. 678733) to FL and CJ.

7

8

9

10

11

12

13

14

15

16

17

18

19

20

21

22

23

24 
1 Declarations of interest

2 None.

3

4

5

6

7

8

9 


\section{References}

2 [1] I. Cross, Music and cognitive evolution, in: R. Dunbar, L. Barrett (Eds.), The Oxford

3 Handbook of Evolutionary Psychology, Oxford University Press, New York, NY, $4 \quad 2007$, pp. 649-667.

5 [2] S.J. Mithen, The Singing Neanderthals: The Origins of Music, Language, Mind, and 6 Body, Weidenfeld \& Nicolson, London, UK, 2005.

7 [3] C. Darwin, The Descent of Man and Selection in Relation to Sex, John Murray, London, $8 \quad$ UK, 1871.

9 [4] B.G. Levman, The genesis of music and language, Ethnomusicology 36(2) (1992) 147$10 \quad 170$.

11 [5] J. Blaking, How Musical Is Man, University of Washington Press, Seattle, WA, 1973.

12 [6] N. Masataka, Pitch characteristics of Japanese maternal speech to infants, J. Child Lang. 19(2) (1992) 213-223.

14 [7] C.E. Snow, Imitativeness: a trait or a skill?, in: G.E. Speidel, K.E. Nelson (Eds.), The 15 Many Faces of Imitation in Language Learning, Springer, New York, NY, 1989, pp. $16 \quad 73-90$.

17 [8] D.J. Hargreaves, R. MacDonald, D. Miell, How do people communicate using music, in:

18 D. Miell, R. MacDonald, D.J. Hargreaves (Eds.), Musical Communication, Oxford 19 University Press, Oxford, UK, 2005, pp. 1-25.

20 [9] S. Koelsch, Brain and Music, John Wiley \& Sons, West Sussex, UK, 2013.

21 [10] S. Koelsch, Towards a neural basis of processing musical semantics, Phys. Life Rev. 22 8(2) (2011) 89-105. 
1 [11] S. Davies, Musical Meaning and Expression, Cornell University Press, New York, NY, 1994.

3 [12] M. Leman, Embodied Music Cognition and Mediation Technology, The MIT Press, Cambridge, MA, 2007.

5 [13] A. Cox, Music and Embodied Cognition: Listening, Moving, Feeling, and Thinking,

$6 \quad$ Indiana University Press, Indiana, IN, 2016.

7 [14] A. Cox, The mimetic hypothesis and embodied musical meaning, Music. Sci. 5(2)

$8 \quad$ (2001) 195-212.

9 [15] N. Steinbeis, S. Koelsch, Understanding the intentions behind man-made products 10 elicits neural activity in areas dedicated to mental state attribution, Cereb. Cortex 19(3) 11 (2009) 619-623.

12 [16] G. Rizzolatti, L. Craighero, The mirror-neuron system, Annu. Rev. Neurosci. 27 (2004) $13 \quad 169-192$.

14 [17] H.M. Hobson, D.V.M. Bishop, The interpretation of mu suppression as an index of mirror neuron activity: past, present and future, Royal Soc. Open Sci. 4(3) (2017).

16 [18] P. Molenberghs, R. Cunnington, J.B. Mattingley, Brain regions with mirror properties: a meta-analysis of 125 human fMRI studies, Neurosci. Biobehav. Rev. 36(1) (2012)

19 [19] M. Iacoboni, M. Dapretto, The mirror neuron system and the consequences of its dysfunction, Nat. Rev. Neurosci. 7(12) (2006) 942-951.

21 [20] M. Iacoboni, R.P. Woods, M. Brass, H. Bekkering, J.C. Mazziotta, G. Rizzolatti,

22 Cortical mechanisms of human imitation, Science 286(5449) (1999) 2526-2528. 
1 [21] S. Caspers, K. Zilles, A.R. Laird, S.B. Eickhoff, ALE meta-analysis of action observation and imitation in the human brain, Neuroimage 50(3) (2010) 1148-1167.

3 [22] P.G. Simos, E. Kavroulakis, T. Maris, E. Papadaki, T. Boursianis, G. Kalaitzakis, H.E. Savaki, Neural foundations of overt and covert actions, Neuroimage 152 (2017) 482496.

6 [23] K. Sakreida, S. Higuchi, C. Di Dio, M. Ziessler, M. Turgeon, N. Roberts, S. Vogt,

7 Cognitive control structures in the imitation learning of spatial sequences and

8 rhythms—an fMRI study, Cereb. Cortex 28(3) (2018) 907-923.

9 [24] D. Corbo, G.A. Orban, Observing others speak or sing activates spt and neighboring

10 parietal cortex, J. Cogn. Neurosci. 29(6) (2017) 1002-1021.

11 [25] S. Hétu, M. Grégoire, A. Saimpont, M.-P. Coll, F. Eugène, P.-E. Michon, P.L. Jackson, 12 The neural network of motor imagery: an ALE meta-analysis, Neurosci. Biobehav. 13 Rev. 37(5) (2013) 930-949.

14 [26] M. Jeannerod, Neural simulation of action: a unifying mechanism for motor cognition, 15 Neuroimage 14(1) (2001) S103-S109.

16 [27] F. Lui, G. Buccino, D. Duzzi, F. Benuzzi, G. Crisi, P. Baraldi, P. Nichelli, C.A. Porro, 17 G. Rizzolatti, Neural substrates for observing and imagining non-object-directed actions, Soc. Neurosci. 3(3-4) (2008) 261-275.

19 [28] R. Jardri, D. Pins, M. Bubrovszky, P. Despretz, J.-P. Pruvo, M. Steinling, P. Thomas, Self awareness and speech processing: an fMRI study, Neuroimage 35(4) (2007) 16451653. 
1 [29] M. Tettamanti, G. Buccino, M.C. Saccuman, V. Gallese, M. Danna, P. Scifo, F. Fazio,

2 G. Rizzolatti, S.F. Cappa, D. Perani, Listening to action-related sentences activates

3 fronto-parietal motor circuits, J. Cogn. Neurosci. 17(2) (2005) 273-281.

4 [30] K. Watkins, T. Paus, Modulation of motor excitability during speech perception: the

5 role of Broca's area, J. Cogn. Neurosci. 16(6) (2004) 978-987.

6 [31] M. Tettamanti, R. Manenti, P.A. Della Rosa, A. Falini, D. Perani, S.F. Cappa, A.

7 Moro, Negation in the brain: modulating action representations, Neuroimage 43(2)

$8 \quad$ (2008) 358-367.

9 [32] V. Gazzola, L. Aziz-Zadeh, C. Keysers, Empathy and the somatotopic auditory mirror 10 system in humans, Curr. Biol. 16(18) (2006) 1824-1829.

11 [33] L.R. Engel, C. Frum, A. Puce, N.A. Walker, J.W. Lewis, Different categories of living 12 and non-living sound-sources activate distinct cortical networks, Neuroimage 47(4) $13 \quad$ (2009) 1778-1791.

14 [34] E. Ricciardi, D. Bonino, L. Sani, T. Vecchi, M. Guazzelli, J.V. Haxby, L. Fadiga, P. 15 Pietrini, Do we really need vision? How blind people "see" the actions of others, J. $16 \quad$ Neurosci. 29(31) (2009) 9719-9724.

17 [35] G. Di Cesare, F. Fasano, A. Errante, M. Marchi, G. Rizzolatti, Understanding the 18 internal states of others by listening to action verbs, Neuropsychologia 89 (2016) 172$19 \quad 179$.

20 [36] M. Ghio, M. Tettamanti, Semantic domain-specific functional integration for action21 related vs. abstract concepts, Brain Lang. 112(3) (2010) 223-232. 
1 [37] P. Molenberghs, R. Cunnington, J.B. Mattingley, Is the mirror neuron system involved in imitation? A short review and meta-analysis, Neurosci. Biobehav. Rev. 33(7) (2009) 975-980.

4 [38] I. Molnar-Szakacs, K. Overy, Music and mirror neurons: from motion to 'e'motion, Soc. Cogn. Affect. Neurosci. 1(3) (2006) 235-241.

6 [39] J. Hou, R. Rajmohan, D. Fang, K. Kashfi, K. Al-Khalil, J. Yang, W. Westney, C.M.

7 Grund, M.W. O'Boyle, Mirror neuron activation of musicians and non-musicians in response to motion captured piano performances, Brain Cogn. 115 (2017) 47-55.

9 [40] B. Haslinger, P. Erhard, E. Altenmüller, U. Schroeder, H. Boecker, A.O. Ceballos-

10 Baumann, Transmodal sensorimotor networks during action observation in

11 professional pianists, J. Cogn. Neurosci. 17(2) (2005) 282-293.

12 [41] J.L. Chen, V.B. Penhune, R.J. Zatorre, Listening to musical rhythms recruits motor regions of the brain, Cereb. Cortex 18(12) (2008) 2844-2854.

14 [42] L.M. McGarry, J.A. Pineda, F.A. Russo, The role of the extended MNS in emotional and nonemotional judgments of human song, Cogn. Affect. Behav. Neurosci. 15(1)

17 [43] G. Hickok, B. Buchsbaum, C. Humphries, T. Muftuler, Auditory-motor interaction revealed by fMRI: speech, music, and working memory in area Spt, J. Cogn. Neurosci. (2015) 32-44. 15(5) (2003) 673-682.

[44] N. Konoike, Y. Kotozaki, H.J. Jeong, A. Miyazaki, K. Sakaki, T. Shinada, M. Sugiura, R. Kawashima, K. Nakamura, Temporal and motor representation of rhythm in frontoparietal cortical areas: an fMRI study, PLoS One 10(6) (2015). 
1 [45] N. Konoike, Y. Kotozaki, S. Miyachi, C.M. Miyauchi, Y. Yomogida, Y. Akimoto, K.

2 Kuraoka, M. Sugiura, R. Kawashima, K. Nakamura, Rhythm information represented

3 in the fronto-parieto-cerebellar motor system, Neuroimage 63(1) (2012) 328-338.

4 [46] S. Koelsch, E. Kasper, D. Sammler, K. Schulze, T. Gunter, A.D. Friederici, Music,

$5 \quad$ language and meaning: brain signatures of semantic processing, Nat. Neurosci. 7(3)

$6 \quad$ (2004) 302-307.

7 [47] L. Zhou, F. Liu, X. Jing, C. Jiang, Neural differences between the processing of

8 musical meaning conveyed by direction of pitch change and natural music in

$9 \quad$ congenital amusia, Neuropsychologia 96 (2017) 29-38.

10 [48] M.M. Bradley, S. Hamby, A. Löw, P.J. Lang, Brain potentials in perception: picture

11 complexity and emotional arousal, Psychophysiology 44(3) (2007) 364-373.

12 [49] I. Peretz, J. Ayotte, R.J. Zatorre, J. Mehler, P. Ahad, V.B. Penhune, B.t. Jutras,

13 Congenital amusia: a disorder of fine-grained pitch discrimination, Neuron 33(2)

$14 \quad$ (2002) 185-191.

15 [50] C. Jiang, V.K. Lim, H. Wang, J.P. Hamm, Difficulties with pitch discrimination

16 influences pitch memory performance: evidence from congenital amusia, PLoS One

$17 \quad 8(10)(2013)$ e 79216.

18 [51] J.M. Foxton, J.L. Dean, R. Gee, I. Peretz, T.D. Griffiths, Characterization of deficits in

19 pitch perception underlying 'tone deafness', Brain 127(4) (2004) 801-810.

20 [52] C. Jiang, J.P. Hamm, V.K. Lim, I.J. Kirk, Y. Yang, Fine-grained pitch discrimination

21 in congenital amusics with Mandarin Chinese, Music Percept. 28(5) (2011) 519-526.

22 [53] K.L. Hyde, I. Peretz, Brains that are out of tune but in time, Psychol. Sci. 15(5) (2004)

$23 \quad 356-360$. 
1 [54] F. Liu, A.D. Patel, A. Fourcin, L. Stewart, Intonation processing in congenital amusia: discrimination, identification and imitation, Brain 133(6) (2010) 1682-1693.

3 [55] P. Loui, F.H. Guenther, C. Mathys, G. Schlaug, Action-perception mismatch in tonedeafness, Curr. Biol. 18(8) (2008) R331-R332.

5 [56] C. Jiang, J.P. Hamm, V.K. Lim, I.J. Kirk, Y. Yang, Processing melodic contour and 6 speech intonation in congenital amusics with Mandarin Chinese, Neuropsychologia 7 48(9) (2010) 2630-2639.

8 [57] A.D. Patel, Music, Language, and the Brain, Oxford University Press, Oxford, UK, 92008.

10 [58] D.T. Vuvan, S. Paquette, G. Mignault Goulet, I. Royal, M. Felezeu, I. Peretz, The 11 Montreal Protocol for Identification of Amusia, Beh. Res. Meth. 50(2) (2018) 662-672.

12 [59] I. Peretz, A.S. Champod, K. Hyde, Varieties of musical disorders, Ann. N. Y. Acad. 13 Sci. 999(1) (2003) 58-75.

14 [60] R.C. Oldfield, The assessment and analysis of handedness: the Edinburgh Inventory, 15 Neuropsychologia 9(1) (1971) 97-113.

16 [61] C. Yan, X. Wang, X. Zuo, Y. Zang, DPABI: Data processing \& analysis for (resting17 state) brain imaging, Neuroinformatics 14(3) (2016) 339-351.

[62] C. Yan, Y. Zang, DPARSF: a MATLAB toolbox for "pipeline" data analysis of resting-state fMRI, Front. Syst. Neurosci. 4(13) (2010).

[63] J.A. Maldjian, P.J. Laurienti, R.A. Kraft, J.H. Burdette, An automated method for neuroanatomic and cytoarchitectonic atlas-based interrogation of fMRI data sets, Neuroimage 19(3) (2003) 1233-1239. 
1 [64] S.B. Eickhoff, T. Paus, S. Caspers, M.-H. Grosbras, A.C. Evans, K. Zilles, K. Amunts, 2 Assignment of functional activations to probabilistic cytoarchitectonic areas revisited, $3 \quad$ Neuroimage 36(3) (2007) 511-521.

4 [65] S.B. Eickhoff, K.E. Stephan, H. Mohlberg, C. Grefkes, G.R. Fink, K. Amunts, K.

5 Zilles, A new SPM toolbox for combining probabilistic cytoarchitectonic maps and

6 functional imaging data, Neuroimage 25(4) (2005) 1325-1335.

7 [66] N.A. Macmillan, C.D. Creelman, Detection Theory: A User's Guide, 2nd ed.,

8 Lawrence Erlbaum Associates, Inc., Mahwah, NJ, 2005.

9 [67] M.J. Hautus, Corrections for extreme proportions and their biasing effects on estimated 10 values of d', Behav. Res. Methods Instrum. Comput. 27(1) (1995) 46-51.

11 [68] M.J. Hautus, A. Lee, Estimating sensitivity and bias in a yes/no task, Br. J. Math. Stat. $12 \quad$ Psychol. 59(2) (2006) 257-273.

13 [69] K. Grill-Spector, R. Henson, A. Martin, Repetition and the brain: neural models of 14 stimulus-specific effects, Trends Cogn. Sci. 10(1) (2006) 14-23.

15 [70] K. Grill-Spector, R. Malach, fMR-adaptation: a tool for studying the functional 16 properties of human cortical neurons, Acta Psychol. (Amst.) 107(1) (2001) 293-321.

17 [71] B.H. Kantowitz, H.L. Roediger III, D.G. Elmes, Experimental Psychology, 10th ed., 18 Cengage Learning, Stamford, CT, 2015.

19 [72] M.R. Leary, Introduction to Behavioral Research Methods, 7th ed., Pearson Education, $20 \quad$ Inc., New York, NY, 2017.

21 [73] T. Chaminade, A.N. Meltzoff, J. Decety, An fMRI study of imitation: action 22 representation and body schema, Neuropsychologia 43(1) (2005) 115-127. 
1 [74] F.P. de Lange, M. Spronk, R.M. Willems, I. Toni, H. Bekkering, Complementary systems for understanding action intentions, Curr. Biol. 18(6) (2008) 454-457.

3 [75] M. Iacoboni, I. Molnar-Szakacs, V. Gallese, G. Buccino, J.C. Mazziotta, G. Rizzolatti, 4 Grasping the intentions of others with one's own mirror neuron system, PLoS Biol. $5 \quad 3(3)(2005)$ e 79.

6 [76] L. Koski, A. Wohlschläger, H. Bekkering, R.P. Woods, M.-C. Dubeau, J.C. Mazziotta, 7 M. Iacoboni, Modulation of motor and premotor activity during imitation of target8 directed actions, Cereb. Cortex 12(8) (2002) 847-855.

9 [77] J. Pa, G. Hickok, A parietal-temporal sensory-motor integration area for the human 10

12 [78] F.C. Binkofski, J. Klann, S. Caspers, On the neuroanatomy and functional role of the

[79] K. Sakreida, I. Effnert, S. Thill, M.M. Menz, D. Jirak, C.R. Eickhoff, T. Ziemke, S.B. inferior parietal lobule and intraparietal sulcus, in: G. Hickok, S.L. Small (Eds.), Neurobiology of Language, Academic Press, San Diego, 2016, pp. 35-47.

(16)
Eickhoff, A.M. Borghi, F. Binkofski, Affordance processing in segregated parietofrontal dorsal stream sub-pathways, Neurosci. Biobehav. Rev. 69 (2016) 89-112.

[80] M. Belyk, P.Q. Pfordresher, M. Liotti, S. Brown, The neural basis of vocal pitch imitation in humans, J. Cogn. Neurosci. 28(4) (2016) 621-635.

[81] J. Jastorff, R.O. Abdollahi, F. Fasano, G.A. Orban, Seeing biological actions in 3D: an fMRI study, Hum. Brain Mapp. 37(1) (2016) 203-219.

22 [82] L.E. Marsh, G. Bird, C. Catmur, The imitation game: effects of social cues on 23 'imitation' are domain-general in nature, Neuroimage 139 (2016) 368-375. 
1 [83] Y. Nan, T.R. Knösche, S. Zysset, A.D. Friederici, Cross-cultural music phrase processing: an fMRI study, Hum. Brain Mapp. 29(3) (2008) 312-328.

[84] A.M. Leaver, J. Van Lare, B. Zielinski, A.R. Halpern, J.P. Rauschecker, Brain activation during anticipation of sound sequences, J. Neurosci. 29(8) (2009) 24772485.

6 [85] R. Harris, B.M. de Jong, Differential parietal and temporal contributions to music

7 perception in improvising and score-dependent musicians, an fMRI study, Brain Res.

$8 \quad 1624(2015) 253-264$.

9 [86] C.S. Pereira, J. Teixeira, P. Figueiredo, J. Xavier, S.L. Castro, E. Brattico, Music and 10 emotions in the brain: familiarity matters, PLoS One 6(11) (2011) e27241.

11 [87] J. Plata Bello, C. Modroño, F. Marcano, J.L. González-Mora, Observation of simple 12 intransitive actions: the effect of familiarity, PLoS One 8(9) (2013) e74485.

13 [88] E. Tulving, H.J. Markowitsch, F.I.M. Craik, R. Habib, S. Houle, Novelty and familiarity activations in PET studies of memory encoding and retrieval, Cereb. Cortex 6(1) (1996) 71-79.

[89] L. Craighero, G. Rizzolatti, The premotor theory of attention, in: L. Itti, G. Rees, J.K. Tsotsos (Eds.), Neurobiology of Attention, Academic Press, Burlington, 2005, pp. 181186.

[90] G. Rizzolatti, L. Craighero, Spatial attention: mechanisms and theories, in: M. Sabourin, F. Craik, M. Robert (Eds.), Advances in Psychological Science, Psychology Press, East Sussex, England, 1998, pp. 171-198. 
1 [91] G. Rizzolatti, L. Riggio, I. Dascola, C. Umiltá, Reorienting attention across the horizontal and vertical meridians: evidence in favor of a premotor theory of attention, Neuropsychologia 25(1) (1987) 31-40.

[92] G. Rizzolatti, L. Riggio, B.M. Sheliga, Space and selective attention, in: C. Umiltà, M. Moscovitch (Eds.), Attention and Performance 15, The MIT Press, Cambridge, MA, 1994, pp. 232-265.

7 [93] D.T. Smith, T. Schenk, The premotor theory of attention: time to move on?,

$8 \quad$ Neuropsychologia 50(6) (2012) 1104-1114.

9 [94] L.M. Ward, Supramodal and modality-specific mechanisms for stimulus-driven shifts 10 of auditory and visual attention, Can. J. Exp. Psychol. 48(2) (1994) 242-59.

11 [95] L. Aziz-Zadeh, M. Iacoboni, E. Zaidel, S. Wilson, J. Mazziotta, Left hemisphere motor 12 facilitation in response to manual action sounds, Eur. J. Neurosci. 19(9) (2004) 2609132612.

14 [96] J.W. Lewis, J.A. Brefczynski, R.E. Phinney, J.J. Janik, E.A. DeYoe, Distinct cortical 15 pathways for processing tool versus animal sounds, J. Neurosci. 25(21) (2005) 5148165158.

17 [97] L. Aziz-Zadeh, L. Koski, E. Zaidel, J. Mazziotta, M. Iacoboni, Lateralization of the 18 human mirror neuron system, J. Neurosci. 26(11) (2006) 2964-2970.

[98] L. Aziz-Zadeh, R.B. Ivry, The human mirror neuron system and embodied representations, in: D. Sternad (Ed.), Progress in Motor Control: A Multidisciplinary Perspective, Springer, Boston, MA, 2009, pp. 355-376. 
1 [99] T.-L. Chou, J.R. Booth, D.D. Burman, T. Bitan, J.D. Bigio, D. Lu, N.E. Cone,

2 Developmental changes in the neural correlates of semantic processing, Neuroimage $329(4)(2006) 1141-1149$.

4 [100] T.-L. Chou, C.-W. Chen, M.-Y. Wu, J.R. Booth, The role of inferior frontal gyrus and 5 inferior parietal lobule in semantic processing of Chinese characters, Exp. Brain Res. $6 \quad$ 198(4) (2009) 465-475.

7 [101] E. Klepousniotou, V.L. Gracco, G.B. Pike, Pathways to lexical ambiguity: fMRI 8 evidence for bilateral fronto-parietal involvement in language processing, Brain Lang. $9 \quad 131(2014) 56-64$.

10 [102] M. Catani, D.K. Jones, D.H. ffytche, Perisylvian language networks of the human 11 brain, Ann. Neurol. 57(1) (2005) 8-16.

12 [103] J. Wang, L. Fan, Y. Zhang, Y. Liu, D. Jiang, Y. Zhang, C. Yu, T. Jiang, 13 Tractography-based parcellation of the human left inferior parietal lobule, Neuroimage 63(2) (2012) 641-652.

15 [104] X. Lu, Y. Sun, H.T. Ho, W.F. Thompson, Pitch contour impairment in congenital 16 amusia: new insights from the Self-paced Audio-visual Contour Task (SACT), PLoS $17 \quad$ One 12(6) (2017) e0179252.

18 [105] C. Jiang, F. Liu, W.F. Thompson, Impaired explicit processing of musical syntax and 19 tonality in a group of Mandarin-speaking congenital amusics, Music Percept. 33(4) $20 \quad$ (2016) 401-413.

21 [106] D. Omigie, M.T. Pearce, L. Stewart, Tracking of pitch probabilities in congenital 22 amusia, Neuropsychologia 50(7) (2012) 1483-1493. 
1 [107] B.R. Zendel, M.-É. Lagrois, N. Robitaille, I. Peretz, Attending to pitch information inhibits processing of pitch information: the curious case of amusia, J. Neurosci. 35(9) (2015) $3815-3824$.

4 [108] J. Ayotte, I. Peretz, K. Hyde, Congenital amusia: a group study of adults afflicted with a music-specific disorder, Brain 125(2) (2002) 238-251.

6 [109] N. Gosselin, S. Paquette, I. Peretz, Sensitivity to musical emotions in congenital 7 amusia, Cortex 71 (2015) 171-182.

8 [110] C. Jiang, F. Liu, P.C.M. Wong, Sensitivity to musical emotion is influenced by tonal $9 \quad$ structure in congenital amusia, Sci. Rep. 7(1) (2017) 7624.

10 [111] M.M. Marin, W.F. Thompson, B. Gingras, L. Stewart, Affective evaluation of 11 simultaneous tone combinations in congenital amusia, Neuropsychologia 78 (2015) $12 \quad 207-220$.

13 [112] K.L. Hyde, R.J. Zatorre, I. Peretz, Functional MRI evidence of an abnormal neural

[114] P. Albouy, J. Mattout, G. Sanchez, B. Tillmann, A. Caclin, Altered retrieval of melodic information in congenital amusia: insights from dynamic causal modeling of MEG data, Front. Hum. Neurosci. 9 (2015) 20. 
1 [115] E. Nagy, H. Compagne, H. Orvos, A. Pal, P. Molnar, I. Janszky, K.A. Loveland, G.

2 Bardos, Index finger movement imitation by human neonates: motivation, learning, 3 and left-hand preference, Pediatr. Res. 58(4) (2005) 749-753.

4 [116] P. Nyström, T. Ljunghammar, K. Rosander, C. von Hofsten, Using mu rhythm 5 desynchronization to measure mirror neuron activity in infants, Dev. Sci. 14(2) (2011) $6 \quad 327-335$.

7 [117] I. Peretz, Musical disorders from behavior to genes, Curr. Dir. Psychol. Sci. 17(5) $8 \quad$ (2008) 329-333.

9 [118] I. Peretz, K.L. Hyde, What is specific to music processing? Insights from congenital 10 amusia, Trends Cogn. Sci. 7(8) (2003) 362-367.

11 [119] I. Peretz, S. Cummings, M.-P. Dubé, The genetics of congenital amusia (tone 12 deafness): a family-aggregation study, Am. J. Hum. Genet. 81(3) (2007) 582-588.

13 [120] D. Drayna, A. Manichaikul, M. de Lange, H. Snieder, T. Spector, Genetic correlates 14 of musical pitch recognition in humans, Science 291(5510) (2001) 1969-1972. 


\section{Figure captions}

2 Fig. 1. Design of the semantic priming paradigm. Melodic sequences with either upward or

3 downward pitch direction changes were used as primes and pictures depicting upward or

4 downward movements were used as targets.

5 Fig. 2. Significant activation areas in controls compared to amusics. The color scale reflects

6 the $t$ value for the contrast between incongruent and congruent conditions for voxels above

7 the FDR-corrected threshold of $p<.05$; the more yellow a pixel is, the higher the $t$ value is.

8

9

10

11

12

13

14

15

16

17

18

19

20

21 